Results Of 253 RRT + patients, $22 \%$ survived ICU and $13 \%$ survived hospital. Of $225 \mathrm{RRT}^{-}$, 81\% survived ICU and 59\% survived hospital (p. On day 1 of admission, RRT + had a higher prevalence of systemic inflammatory response syndrome (76\% vs 62\%), ChildPugh (12 vs 11), MELD (32 vs 17) and SOFA scores (13 vs 11) (pEven when the renal component was removed from the SOFA score (SOFAminusRENAL), RRT + had higher scores than RRT- (9 vs 7) (p. $23 \%$ of RRT + commenced RRT after day 3 of admission; this did not affect ICU or hospital survival compared to those that commenced RRT before day 3. RRT+ survivors required less ventilation (39\% vs $93 \%$ ) and vasopressors ( $52 \%$ vs $89 \%$ ) than RRT + non-survivors and had lower Child-Pugh (12 vs 13), SOFA (12 vs 14) and SOFAminusRENAL ( 8 vs 10) scores ( $p=1$ of admission. 23 patients required RRT but no other organ support, 18/23 (78\%) survived to ICU discharge and 11/23 (48\%) to hospital discharge.

Conclusion The extent of MODS, rather than requirement of RRT per se, dictates poor prognosis in cirrhotics needing RRT in ICU. Requirement for RRT should not preclude admission to ICU, rather, prognostication should take into account other elements of MODS; in particular a concomitant requirement for circulatory and respiratory support.

Competing interests None declared.

\section{PWE-276 VALIDATION OF FIBROSCAN AS A SCREENING TOOL FOR CYSTIC FIBROSIS ASSOCIATED LIVER DISEASE IN AN ADULT POPULATION}

doi:10.1136/gutjnl-2012-302514d.276

${ }^{1} \mathrm{R}$ E Swann, * 'Z Mustafa, ${ }^{2} \mathrm{E}$ Ross, ${ }^{1} \mathrm{M}$ Priest. 'Department of Gastroenterology, NHSGGC Gartnavel General Hospital, Glasgow, UK; ${ }^{2}$ Department of Respiratory, NHSGGC Gartnavel General Hospital, Glasgow, UK

Introduction Cystic fibrosis (CF) associated liver disease affects up to $41 \%$ of CF sufferers and may progress to cirrhosis with associated complications. ${ }^{1}$ Diagnosis is frequently made without biopsy. A recent study evaluated the use of fibroscan in diagnosis of $\mathrm{CF}$ associated liver disease (CFLD) in the paediatric context, however there are now many adult patients with cystic fibrosis, therefore we aimed to validate the ability of fibroscan to assess liver disease severity in an adult CF population. ${ }^{23}$

Methods We recruited a cohort of adult CF patients diagnosed in the paediatric setting with CFLD and a control cohort with CF but no clinical or biochemical evidence of liver disease. All patients were assessed for clinical, radiological or biochemical evidence of liver disease by the authors and underwent a fibroscan. Fibroscan results were correlated with clinical evidence of liver disease.

Results We recruited 20 patients, 11 with normal liver biochemistry, no clinical evidence of liver disease and normal liver ultrasound scans (NLF group) and nine with a historical diagnosis of CF associated liver disease. Six of these had abnormal liver function tests but no clinical, radiological or endoscopic evidence of cirrhosis (Intermediate-I) and three patients had significant liver disease with evidence of portal hypertension at endoscopy (SLD). Correlation of fibroscan result with clinical group was performed. The difference between the SLD group and the NLF group was significant $(p=0.002)$ by the Mann-Witney U test. A ROC analysis suggested a cut-off of $11.2 \mathrm{kPa}$ for cirrhotic CFLD as having the highest accuracy. However this requires further validation with a larger cohort of patients. There was strong correlataion of fibroscan reading with APRI score with an $\mathrm{R}^{2}$ value of 0.757 .

Conclusion Fibroscan correlates well with clinical assessment of CFLD severity in our cohort of adult CF patients and may help clarify diagnosis. We continue to recruit patients and hope to determine appropriate cut-off values for further investigation.

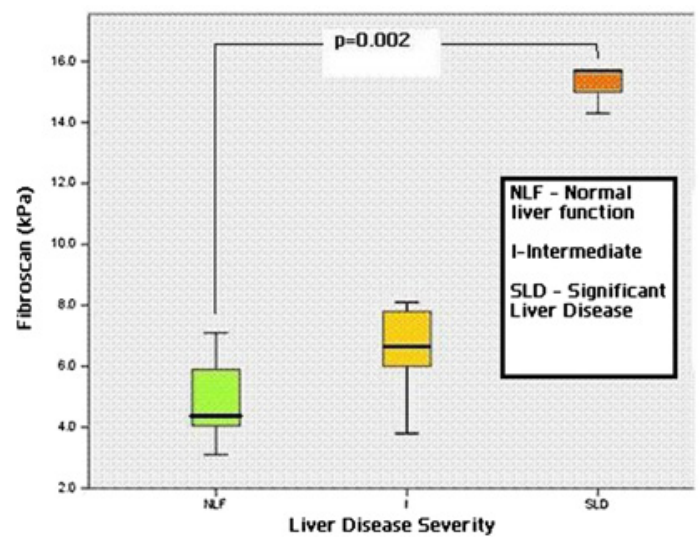

Abstract PWE-276 Figure 1 Fibroscan $(\mathrm{kPa})$ by liver disease severity.

Competing interests None declared.

\section{REFERENCE}

Lamireau T, Monnereau S, Martin S, et al. J Hepatol 2004;41:920-5.

Witters P, De Boeck K, Dupont L, et al. J Cyst Fibros 2009:8:392-9.

Leonard A, Menten R, Clapuyt P, et al. J Cyst Fibros 2008;7(Suppl 2):S82.

\section{PWE-277 FRACTURE PREVALENCE AND VITAMIN D STATUS IN PRIMARY BILIARY CIRRHOSIS: THE LEICESTERSHIRE EXPERIENCE}

doi:10.1136/gutjnl-2012-302514d.277

${ }^{1} \mathrm{R}$ Krishnamoorthy, ${ }^{* 2} \mathrm{~A}$ Grant, ${ }^{3} \mathrm{P}$ Sheldon, ${ }^{2} \mathrm{~T}$ Delahooke. ${ }^{1}$ Digestive Diseases Centre, University Hospitals Leicester NHS Trust, Leicester, UK; ${ }^{2}$ Department of Hepatology, University Hospitals Leicester NHS Trust, Leicester, UK; ${ }^{3}$ Department of Rheumatology, University Hospitals Leicester NHS Trust, Leicester, UK

Introduction Metabolic bone disease is a recognised complication in Primary Biliary Cirrhosis (PBC) and increases the risk of developing fractures. Although osteoporosis is the major contributor, Vitamin D (25- hydroxy- vitamin- D3) deficiency due to fat-soluble vitamin malabsorption is also a contributing factor for bone disease in PBC. Our objective was to assess the prevalence of fractures and vitamin $\mathrm{D}$ deficiency in PBC patients.

Methods Patients with diagnosed with PBC between the years 1994 and 2011 were retrospectively identified from the hepatology outpatients. Fracture data were collected from the x-ray reports in the radiology software. Biochemical data including AMA titres and Vitamin D status were retrospectively identified and entered using the pathology database. The grading for Vitamin D levels were as follows: severely deficient- $20 \mathrm{mg} / \mathrm{l}$ or $>50 \mathrm{nmol} / \mathrm{l}$. Available Bone Mineral Density (BMD) data in patients who had a Dual-emission $\mathrm{x}$-ray absorptiometry (DEXA) scan was studied.

Results Among 209 patients (179 female, median age 68 years, 168 AMA positive with median AMA titre of 1 in 256) with PBC, 27 patients $(12.9 \%$, 25 females, median age 74$)$ had sustained a fracture during their clinical course. 33 fracture episodes were identified. Femur/hip fractures were the commonest (9/33, 27\%), followed by hand $(5 / 33,15 \%)$. DEXA scans were performed in 39 patients, with a median $\mathrm{T}$ score of -2.2 . Vitamin D levels were available in 91 patients (44\%), the levels being adequate in only 27 patients (29.6\%), reflecting the magnitude of the Vitamin D status. 38 patients were insufficient $(41.7 \%), 17$ were deficient $(18.6 \%)$ and 9 were severely deficient (0.09\%).

Conclusion Fracture prevalence and vitamin $\mathrm{D}$ deficiency is high in PBC patients. Assessing Vitamin D status is a useful measure to improve bone health and reduce the burden of metabolic bone disease. 
Competing interests None declared.

\section{REFERENCE}

1. Solaymani-Dodaran $\mathbf{M}$, Card TR, Aithal GP, et al. Fracture risk in people with primary biliary cirrhosis: a population-based cohort study. Gastroenterology 2006;131:1752.

\section{PWE-278 DOES N-ACETYL CYSTEINE MODULATE RENAL FUNCTION AFTER MAJOR HEPATECTOMY?}

doi:10.1136/gutjnl-2012-302514d.278

R Lochan, * A Boukas, S Robinson, R Saif, D Manas, S White. HPB\& Liver Transplantation Surgery, FRH, Newcastle Upon Tyne, UK

Introduction Acute kidney injury or deterioration in pre-existing renal dysfunction following major hepatectomy is an important cause of morbidity and mortality. N-acetyl cysteine has been extensively utilised as a reno-protective agent to ameliorate contrast induced nephropathy. The aim of this study was to investigate its potential use for preserving renal function following major hepatectomy.

Methods A prospective study to compare the impact of N-Acetyl cysteine (NAC) on liver function after hepatectomy was undertaken in our Unit from 2004 to 2010. A cohort of 44 patients received perioperative NAC $(10 \mathrm{~g} / 24 \mathrm{~h})$ for 5 days and were compared to a further cohort of 44 patients (matched for the extent of liver resection-all more than three segments), gender, age and chemotherapy use) who did not. Post-operative renal function was evaluated. Other variables known to influence renal function included associated co-morbidity, drugs, intra-operative parameters (eg, CVP and blood loss). In addition to calculating the renal risk index as described by Clavien et al. We also examined other outcome measures such as duration of renal support and long-term renal specific outcomes.

Results There were three patients with known CKD in the NAC group as compared to one in the control group. Mean (SD) preoperative creatinine levels [93.9 (20.5), 92.5 (19.3) p=0.7], BMI [26.9 (14.5), 28.9 (13.1) $\mathrm{p}=0.3$ ], operating time (h) [6 (1.9), 5.5 (1.7), $p=0.9]$. The mean renal risk index were also similar between the NAC and control group. Mean (SD) creatinine rise (delta creatinine) between days 1 and 5 in the post-operative period, were also similar between the 2 groups [NAC 43.8 (82.9) vs Control 49.2 (63.5), $\mathrm{p}=0.72]$. Renal support in the form of CVVH was needed in three patients in the study group vs one in the control group (all known to have pre-existing CKD). All these patients went back to their preoperative renal status at 3-months follow-up.

Conclusion In this study NAC does not appear to be reno-protective in patients with known renal impairment who require a major liver resection. Its presumed renoprotective role in those at "high risk" needs to be further assessed in an adequately powered RCT.

Competing interests None declared.

\section{PWE-279 THE HEPATIC EFFECTS OF A HIGH FRUCTOSE VS A HIGH GLUCOSE DIET IN HEALTHY OVERWEIGHT MEN}

doi:10.1136/gutjnl-2012-302514d.279

${ }^{1} \mathrm{R}$ D Johnston, ${ }^{*} \mathrm{M}$ C Stephenson, ${ }^{3} \mathrm{H}$ Crossland, ${ }^{3} \mathrm{~S} \mathrm{M}$ Cordon, ${ }^{3} \mathrm{M}$ A Taylor, ${ }^{1} \mathrm{G}$ P Aithal, ${ }^{3}$ I A Macdonald. 'Nottingham Digestive Diseases Centre, National Institute for Health Research Biomedical research Unit, University of Nottingham, Nottingham, UK; ${ }^{2}$ Sir Peter Mansfield Magnetic Resonance Centre, University of Nottingham, Nottingham, UK; ${ }^{3}$ School of Biomedical Sciences, University of Nottingham, Nottingham, UK

Introduction A high intake of fructose has been implicated in NAFLD aetiology. As the majority of dietary fructose originates from sucrose it remains uncertain if these observations are fructose-specific.
Infrequent and inconsistent differences in hepatic metabolism have been shown with a high intake of fructose or glucose, the constituents of sucrose. ${ }^{12}$ There is no prior comparative data in healthy overweight men, and none in the isoenergetic state.

Methods 32 healthy, centrally overweight males were randomised to two periods each of 2 weeks of either a high fructose or glucose intake in a non-crossover fashion. Isoenergetic status was maintained by providing foodstuffs during the first period, followed by a 6 -week washout and then a second period of ad libitum overfeeding. The sugars contributed $25 \%$ of predicted total energy requirements, and were consumed 4 times a day dissolved in water. The primary outcome was hepatic triglyceride content $\left({ }^{1} \mathrm{H}\right.$ MRS), with further assessments of calf lipid ( ${ }^{1} \mathrm{H}$ MRS), deuterated glucose hyperinsulinaemic euglycaemic clamps, and indirect calorimetry. Outcomes assessed by Student $t$ test.

Results The groups were well matched at study entry. Overall the subjects' mean age was 34 years, BMI $29.4 \mathrm{~kg} / \mathrm{m}^{2}$ and daily dose of sugars was $217 \mathrm{~g}$. The changes in the primary measures are shown in the Abstract PWE-279 table 1 below. There were no changes during the energy balanced period. With energy and monosaccharide overfeeding weight, serum triglycerides, liver lipid and biochemistry increased significantly, but to a similar extent in both groups. Further to this the groups did not differ in terms of satiety, whole body oxidative profile, hepatic insulin resistance, calf lipid, or renal function.

Abstract PWE-279 Table 1

\begin{tabular}{llccccl}
\hline & $\begin{array}{l}\text { Energy } \\
\text { balance }\end{array}$ & $\begin{array}{l}\text { Fructose, } \\
\text { mean }\end{array}$ & SD & $\begin{array}{l}\text { Glucose, } \\
\text { mean }\end{array}$ & SD & $\begin{array}{l}\text { Sig between, } \\
\text { groups }\end{array}$ \\
\hline Weight $(\mathrm{kg})$ & Iso & -0.26 & 0.92 & 0.12 & 0.70 & 0.62 \\
& Excess & $1.03^{*}$ & 1.37 & $0.57^{*}$ & 1.00 & 0.29 \\
Hepatic lipid (\%) & Iso & 0.30 & 2.20 & -0.05 & 2.13 & 0.65 \\
& Excess & $1.70^{*}$ & 2.60 & $2.05^{*}$ & 2.89 & 0.73 \\
ALT (U/l) & Iso & -4.0 & 7.9 & -2.9 & 6.5 & 0.67 \\
& Excess & $5.8^{*}$ & 8.7 & 4.1 & 9.8 & 0.62 \\
Triglyceride (mmol/l) & Iso & -0.07 & 0.35 & 0.13 & 0.74 & 0.35 \\
& Excess & 0.36 & 0.75 & $0.33^{* *}$ & 0.39 & 0.91
\end{tabular}

Absolute change in parameters during intervention ${ }^{*} \mathrm{p}<0.05,{ }^{* *} \mathrm{p}<0.01$ as compared to baseline values in that group).

Conclusion There were no differences between a high fructose and glucose diet in relation to hepatic lipids or biochemistry. The changes during the overfeeding period were strongly associated with changes in weight, reinforcing the interpretation that these were an energy, as opposed to a nutrient, specific effect.

Clinical trial registration number NCT01050140.

Competing interests None declared.

\section{REFERENCES}

1. Stanhope KL, Schwarz JM, Keim NL, et al. Consuming fructose-sweetened, not glucose-sweetened, beverages increases visceral adiposity and lipids and decreases insulin sensitivity in overweight/obese humans. J Clin Invest 2009:119:1322-34.

2. Ngo Sock ET, Lê KA, Ith M, et al. Effects of a short-term overfeeding with fructose or glucose in healthy young males. Br J Nutr 2010;103:939-43.

\section{PWE-280 FERROPORTIN DISEASE-AN IMPORTANT CAUSE OF IRON OVERLOAD}

doi:10.1136/gutjnl-2012-302514d.280

${ }^{1} \mathrm{R}$ Mahmood, ${ }^{*}{ }^{2} \mathrm{M}$ Labib, ${ }^{1} \mathrm{~N}$ C Fisher. ${ }^{1}$ Department of Gastroenterology, Russells Hall Hospital, Dudley, UK; ${ }^{2}$ Russells Hall Hospital, Dudley, UK

Introduction Ferroportin disease is a recently characterised cause of iron overload. Unlike hereditary haemochromatosis it involves 